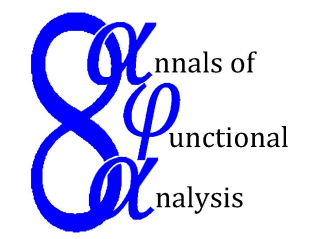

Ann. Funct. Anal. 6 (2015), no. 4, 206-214

http://doi.org/10.15352/afa/06-4-206

ISSN: 2008-8752 (electronic)

http://projecteuclid.org/afa

\title{
THE GENERALIZED VON NEUMANN-JORDAN CONSTANT AND NORMAL STRUCTURE IN BANACH SPACES
}

\author{
XI WANG ${ }^{1 *}$, YUNAN CUI ${ }^{2}$ AND CHIPING ZHANG ${ }^{1}$
}

Communicated by J. Esterle

\begin{abstract}
Recently, a new geometric constant called generalized von NeumannJordan constant was introduced. In this paper, the relationships between above constant and generalized García-Falset coefficient are given. In terms of this constant, the lower bounds for the weakly convergent sequence coefficient of a Banach space $X$ are also shown. Moreover, some sufficient conditions which imply normal structure and uniform normal structure are presented.
\end{abstract}

\section{INTRODUCTION AND PRELIMINARIES}

Let $X$ be a real Banach space. For a nonempty bounded subset $C$ of $X$, denote that

$$
\operatorname{diam}(C):=\sup \{\|x-y\|: x, y \in C\} \text { and } \operatorname{rad}(C):=\inf _{x \in C} \sup \{\|x-y\|: y \in C\} .
$$

We say that $X$ has normal structure if every bounded closed convex subset $C$ of $X$ with $\operatorname{diam}(C)>0$, verifies $\operatorname{rad}(C)<\operatorname{diam}(C) . X$ is said to have uniform normal structure if there exists $c \in(0,1)$ such that, for all bounded closed convex subsets $C$ of $X$ with $\operatorname{diam}(C)>0$, the inequality $\operatorname{rad}(C)<c \operatorname{diam}(C)$ holds.

A Banach space $X$ is said to have the fixed point property if every nonexpansive mapping $T: C \rightarrow C$, i.e.,

$$
\|T x-T y\| \leq\|x-y\|, \forall x, y \in C
$$

acting on a nonempty bounded closed and convex subset $C$ of $X$ has a fixed point. It was proved by Kirk [9] that every reflexive Banach space with normal structure

Date: Received: Apr. 2, 2015; Accepted: Jun. 1, 2015.

* Corresponding author.

2010 Mathematics Subject Classification. Primary 46B20; Secondary 47H10.

Key words and phrases. Generalized von Neumann-Jordan constant, generalized GarcíaFalset coefficient, fixed point property, weakly convergent sequence coefficient, normal structure. 
has the fixed point property. Many geometrical properties of Banach spaces implying normal structure and uniform normal structure have been studied.

The von Neumann-Jordan constant was defined by Clarkson as follows:

$$
C_{N J}(X):=\sup \left\{\frac{\|x+y\|^{2}+\|x-y\|^{2}}{2\left(\|x\|^{2}+\|y\|^{2}\right)}: x, y \in X, \text { not both zero }\right\} .
$$

The weakly convergent sequence coef ficient of $X, W C S(X)$, was defined in [3] as the supremum of the set of all numbers $M$ with the property that for each weakly convergent sequence $\left(x_{n}\right)$, there is some $y$ in the closed convex hull of the sequence such that

$$
M \limsup _{n \rightarrow \infty}\left\|x_{n}-y\right\| \leq \lim _{n \rightarrow \infty} \sup \left\{\left\|x_{i}-x_{j}\right\|: i, j \geq n\right\} .
$$

It is clear that $1 \leq W C S(X) \leq 2$ and it is known that $X$ has weak normal structure if $W C S(X)>1[3]$.

The coefficient $R(a, X)$, which is a generalized García-Falset coefficient $R(X)$ [5], is introduced by Benavides [2]: For a given $a \geq 0$,

$$
R(a, X):=\sup \left\{\liminf _{n \rightarrow \infty}\left\|x_{n}+x\right\|\right\},
$$

where the supremum is taken over all $x \in X$ with $\|x\| \leq a$ and all weakly null sequences in the unit ball $B_{X}$ such that $\lim _{n, m ; n \neq m}\left\|x_{n}-x_{m}\right\| \leq 1$. It is clear that $R(1, X)=R(X)$. Benavides [2] also defined the coefficient $M(X)$ by

$$
M(X):=\sup \left\{\frac{1+a}{R(a, X)}: a \geq 0\right\} .
$$

The coefficient $R W(a, X)$ was introduced in [6]: For each $a \geq 0$,

$$
R W(a, X):=\sup \left\{\min \left\{\liminf _{n \rightarrow \infty}\left\|x_{n}+x\right\|, \liminf _{n \rightarrow \infty}\left\|x_{n}-x\right\|\right\}\right\},
$$

where the supremum is taken over all $x \in X$ with $\|x\| \leq a$ and all weakly null sequences in the unit ball $B_{X}$. It was proved that for any Banach space $X$, the inequality $R W(a, X) \geq R(a, X)$ holds [11].

Recall that

$$
\rho_{X}^{\prime}(0):=\lim _{t \rightarrow 0^{+}} \frac{\rho_{X}(t)}{t},
$$

where $\rho_{X}[0, \infty) \rightarrow[0, \infty)$ is the modulus of smoothness of $X$ defined by

$$
\rho_{X}(t):=\sup \left\{\frac{\|x+t y\|+\|x-t y\|}{2}-1: x, y \in B_{X}\right\} \text {. }
$$

The coefficient $\mu(X)$, defined by the infimum of the set of real numbers $r>0$ such that

$$
\limsup _{n \rightarrow \infty}\left\|x+x_{n}\right\| \leq r \limsup _{n \rightarrow \infty}\left\|x-x_{n}\right\|
$$

for all $x \in X$ and all weakly null sequences $\left(x_{n}\right)$ in $X$. It was proved that if the condition $\rho_{X}^{\prime}(0)<\frac{1}{\mu(X)}$ holds, then $X$ has normal structure [11].

The modulus of convexity of $X$ is the function $\delta_{X}:[0,2] \rightarrow[0,1]$ defined by

$$
\delta_{X}(\varepsilon):=\inf \left\{1-\left\|\frac{x+y}{2}\right\|: x, y \in B_{X},\|x-y\| \geq \varepsilon\right\} .
$$


The characteristic of convexity of $X$ is the number

$$
\varepsilon_{0}(X):=\sup \left\{\varepsilon: \delta_{X}(\varepsilon)=0\right\} .
$$

In this paper, some relationships between the generalized von Neumann-Jordan constant and generalized García-Falset coefficient are given. The lower bounds for the weakly convergent sequence coefficient of a Banach space $X$ are also shown. Moreover, some sufficient conditions for normal structure and uniform normal structure are presented.

\section{THE CONSTANT AND THE FIXED POINT PROPERTY}

Recently, Cui, Huang, Hudzik and Kaczmarek [4] introduced a new geometric constant $C_{N J}^{(p)}(X)$ called generalized von Neumann-Jordan constant, defined by

$$
C_{N J}^{(p)}(X):=\sup \left\{\frac{\|x+y\|^{p}+\|x-y\|^{p}}{2^{p-1}\left(\|x\|^{p}+\|y\|^{p}\right)}: x, y \in X,(x, y) \neq(0,0)\right\},
$$

where $1 \leq p<\infty$.

The parametrized formula of this constant is the following

$$
C_{N J}^{(p)}(X)=\sup \left\{\frac{\|x+t y\|^{p}+\|x-t y\|^{p}}{2^{p-1}\left(1+t^{p}\right)}: x, y \in S_{X}, 0 \leq t \leq 1\right\},
$$

where $1 \leq p<\infty$.

It was proved that the generalized von Neumann-Jordan constant satisfies the inequality $C_{N J}^{(p)}(X) \leq 2$, and that Banach space $X$ is uniformly non-square if and only if $C_{N J}^{(p)}(X)<2[4]$.

Next, we will show the relationships between the constant $C_{N J}^{(p)}(X)$ and the coefficient $R(a, X)$, and we will obtain a sufficient condition for the fixed point property.

Proposition 2.1. Let $X$ be a Banach space. For any $a \geq 0$,

$$
C_{N J}^{(p)}(X) \geq \frac{R(a, X)^{p}}{2^{p-2}\left(1+a^{p}\right)}
$$

Proof. Let $a \geq 0$. For any weakly null sequence $\left(x_{n}\right)$ in $B_{X}$ and any $x \in X$ with $\|x\| \leq a$, by the definition of $C_{N J}^{(p)}(X)$, we have,

$$
C_{N J}^{(p)}(X) \geq \frac{\left\|x_{n}+x\right\|^{p}+\left\|x_{n}-x\right\|^{p}}{2^{p-1}\left(\left\|x_{n}\right\|^{p}+\|x\|^{p}\right)} \geq \frac{\min \left\{\left\|x_{n}+x\right\|^{p},\left\|x_{n}-x\right\|^{p}\right\}}{2^{p-2}\left(\left\|x_{n}\right\|^{p}+\|x\|^{p}\right)}
$$

Therefore,

$$
C_{N J}^{(p)}(X) \geq \frac{R W(a, X)^{p}}{2^{p-2}\left(1+a^{p}\right)}
$$

and since the inequality $R W(a, X) \geq R(a, X)$ holds, we obtain

$$
C_{N J}^{(p)}(X) \geq \frac{R(a, X)^{p}}{2^{p-2}\left(1+a^{p}\right)}
$$


Proposition 2.2. If $X$ is a Banach space, then

$$
C_{N J}^{(p)}(X) \geq \frac{(R(X))^{p}\left(1+\frac{1}{(\mu(X))^{p}}\right)}{2^{-p}} .
$$

Proof. For any $\varepsilon>0$, there exists $x \in S_{X}$ and $\left(x_{n}\right)$ in $B_{X}$ such that

$$
\liminf _{n \rightarrow \infty}\left\|x_{n}+x\right\| \geq R(X)-\varepsilon \text {. }
$$

We may extract a subsequence, still denoted by $\left(x_{n}\right)$, such that $\liminf _{n \rightarrow \infty} \|$ $x_{n}-x \|$ exists and

$$
\liminf _{n \rightarrow \infty}\left\|x_{n}+x\right\| \geq R(X)-\varepsilon
$$

So we obtain,

$$
\begin{aligned}
2^{p} C_{N J}^{(p)}(X) & \geq \liminf _{n \rightarrow \infty}\left\|x_{n}+x\right\|^{p}+\liminf _{n \rightarrow \infty}\left\|x_{n}-x\right\|^{p} \\
& \geq \liminf _{n \rightarrow \infty}\left\|x_{n}+x\right\|^{p}\left(1+\frac{1}{(\mu(X))^{p}}\right) \\
& \geq(R(X)-\varepsilon)^{p}\left(1+\frac{1}{(\mu(X))^{p}}\right) .
\end{aligned}
$$

By the arbitrariness of $\varepsilon>0$, we get $C_{N J}^{(p)}(X) \geq \frac{(R(X))^{p}\left(1+\frac{1}{(\mu(X))^{p}}\right)}{2^{-p}}$.

As a consequence of the preceding proposition, we have the following corollary.

Corollary 2.3. If $C_{N J}^{(p)}(X)<1+\frac{1}{(\mu(X))^{p}}$, then $R(X)<2$, that is, such a Banach space $X$ has the fixed point property.

3. The generalized von Neumann-Jordan COnstant and normal STRUCTURE

Now we show some inequalities on the generalized von Neumann-Jordan constant, and the constants $\varepsilon_{0}(X)$ and $\rho_{X}^{\prime}(0)$. By these inequalities we obtain some sufficient conditions which imply normal structure.

Proposition 3.1. For any Banach space $X$, we have the following:

(1) $C_{N J}^{(p)}(X) \geq 1+\frac{\varepsilon_{0}(X)^{p}}{2^{p}}$,

(2) $C_{N J}^{(p)}(X) \geq 1+\rho_{X}^{\prime}(0)^{p}$.

Proof. (1)Let $\varepsilon \in[0,2]$. Suppose that there exists $x, y \in B_{X}$ such that $\|x-y\| \geq$ $\varepsilon$. Then

which is equivalent to

$$
C_{N J}^{(p)}(X) \geq \frac{\|x+y\|^{p}+\|x-y\|^{p}}{2^{p-1}\left(\|x\|^{p}+\|y\|^{p}\right)} \geq \frac{\|x+y\|^{p}+\varepsilon^{p}}{2^{p}}
$$

$$
1-\left\|\frac{x+y}{2}\right\| \geq 1-\sqrt[p]{C_{N J}^{(p)}(X)-\left(\frac{\varepsilon}{2}\right)^{p}} .
$$

By the definition of $\delta_{X}$, we get

$$
\delta_{X}(\varepsilon) \geq 1-\sqrt[p]{C_{N J}^{(p)}(X)-\left(\frac{\varepsilon}{2}\right)^{p}}
$$


that is,

We conclude that

$$
C_{N J}^{(p)}(X) \geq\left(\frac{\varepsilon}{2}\right)^{p}+\left(1-\delta_{X}(\varepsilon)\right)^{p}
$$

$$
C_{N J}^{(p)}(X) \geq \sup \left\{\left(\frac{\varepsilon}{2}\right)^{p}+\left(1-\delta_{X}(\varepsilon)\right)^{p}: \varepsilon \in[0,2]\right\}
$$

and in particular, by $\varepsilon_{0}(X)=2\left(1-\lim _{\varepsilon \rightarrow 2} \delta_{X}(\varepsilon)\right)($ see $[7])$,

$$
C_{N J}^{(p)}(X) \geq \lim _{\varepsilon \rightarrow 2^{-}}\left(\left(\frac{\varepsilon}{2}\right)^{p}+\left(1-\delta_{X}(\varepsilon)\right)^{p}\right)=1+\left(\frac{\varepsilon_{0}(X)^{p}}{2^{p}}\right) .
$$

(2) $\mathrm{By}(\mathrm{i})$, and $\varepsilon_{0}\left(X^{*}\right)=2 \rho_{X}^{\prime}(0)$ (the consequence of Lindenstrauss' formulae [10]), we obtain that

$$
C_{N J}^{(p)}(X)=C_{N J}^{(p)}\left(X^{*}\right) \geq 1+\left(\frac{\varepsilon_{0}\left(X^{*}\right)^{p}}{2^{p}}\right)=1+\rho_{X}^{\prime}(0)^{p} .
$$

Corollary 3.2. Let $X$ be a Banach space. If $C_{N J}^{(p)}(X)<1+\frac{1}{\mu(X)^{p}}$, then $\rho_{X}^{\prime}(0)<$ $\frac{1}{\mu(X)}$.

Lemma 3.3. [11] Let $X$ be a Banach space. IF $\left(x_{n}\right)$ be a weakly null sequence in $S_{X}$ such that $\lim _{n, m \rightarrow \infty, n \neq m}\left\|x_{n}-x_{m}\right\|=: d$ exists, then there exist $\left(u_{n}\right),\left(v_{n}\right)$ and $\left(w_{n}\right)$, weakly null sequence in $S_{X}$ and $\left(f_{n}\right)$ and $\left(g_{n}\right)$, sequences in $S_{X^{*}}$ for which

$$
\begin{gathered}
\lim _{n \rightarrow \infty} f_{n}\left(-u_{n}\right)=\lim _{n \rightarrow \infty} g_{n}\left(u_{n}\right)=\frac{1}{d}, \\
\lim _{n \rightarrow \infty} f_{n}\left(v_{n}\right) \geq \frac{a}{R(a, X)}, \\
\lim _{n \rightarrow \infty} g_{n}\left(v_{n}\right) \geq \frac{1}{R(a, X) d}
\end{gathered}
$$

and

$$
\min \left\{\lim _{n \rightarrow \infty} f_{n}\left(w_{n}\right), \lim _{n \rightarrow \infty} g_{n}\left(w_{n}\right)\right\} \geq \frac{1}{\mu(X) d} .
$$

Relationships between the generalized von Neumann-Jordan constant, the weakly convergent sequence coefficient, the coefficient $R(a, X)$ and the coefficient $M(X)$ is given in the following proposition.

\section{Proposition 3.4.}

(1) $C_{N J}^{(p)}(X) \geq \frac{1}{W C S(X)^{p}}+\sup \left\{\frac{1}{2^{p} R(a, X)^{p}}\left(a+\frac{1}{W C S(X)}\right)^{p}: a \geq 0\right\}$.

(2) $W C S(X)^{p} \geq \frac{1+\left(\frac{M(X)}{2}\right)^{p}}{C_{N J}^{(p)}(X)}$.

Proof. (1) Let $\left(x_{n}\right)$ be a weakly null sequence in $S_{X}$ such that $\lim _{n, m \rightarrow \infty} \| x_{n}-$ $x_{m} \|=d$ exists. Put $a \geq 0$. By Lemma3.3, there exist weakly null sequence $\left(u_{n}\right)$ and $\left(v_{n}\right)$ in $S_{X}$, sequence $\left(f_{n}\right)$ and $\left(g_{n}\right)$ in $S_{X^{*}}$, for which

$$
\lim _{n \rightarrow \infty} f_{n}\left(-u_{n}\right)=\lim _{n \rightarrow \infty} g_{n}\left(u_{n}\right)=\frac{1}{d}
$$




$$
\lim _{n \rightarrow \infty} f_{n}\left(v_{n}\right) \geq \frac{a}{R(a, X)}
$$

and

$$
\lim _{n \rightarrow \infty} g_{n}\left(v_{n}\right) \geq \frac{1}{R(a, X) d}
$$

For each $n \geq 1,\left\|f_{n}-g_{n}\right\| \geq f_{n}\left(-u_{n}\right)+g_{n}\left(u_{n}\right)$ and $\left\|f_{n}+g_{n}\right\| \geq f_{n}\left(v_{n}\right)+g_{n}\left(v_{n}\right)$, so

$$
\liminf _{n \rightarrow \infty}\left\|f_{n}-g_{n}\right\| \geq \frac{2}{d}
$$

and

$$
\liminf _{n \rightarrow \infty}\left\|f_{n}+g_{n}\right\| \geq \frac{1}{R(a, X)}\left(a+\frac{1}{d}\right) .
$$

Since, for each $n \geq 1$,

$$
C_{N J}^{(p)}(X)=C_{N J}^{(p)}\left(X^{*}\right) \geq \frac{\left\|f_{n}+g_{n}\right\|^{p}+\left\|f_{n}-g_{n}\right\|^{p}}{2^{p}}
$$

we obtain

$$
C_{N J}^{(p)}(X) \geq \frac{1}{d^{p}}+\frac{1}{2^{p} R(a, X)^{p}}\left(a+\frac{1}{d}\right)^{p}
$$

By the equivalent expression of $W C S(X)[1]$, we conclude

$$
C_{N J}^{(p)}(X) \geq \frac{1}{W C S(X)^{p}}+\frac{1}{2^{p} R(a, X)^{p}}\left(a+\frac{1}{W C S(X)}\right)^{p} .
$$

Inequality (2) is a consequence of (i): for all $a>0$,

$$
\begin{aligned}
C_{N J}^{(p)}(X) & \geq \frac{1}{W C S(X)^{p}}+\frac{1}{2^{p} R(a, X)^{p}}\left(a+\frac{1}{W C S(X)}\right)^{p} \\
& \geq \frac{1}{W C S(X)^{p}}\left(1+\left(\frac{1+a}{2 R(a, X)}\right)^{p} .\right.
\end{aligned}
$$

Thus

$$
W C S(X)^{p} \geq \frac{1+\frac{\sup \left\{\frac{1+a}{R(a, X)}: a \geq 0\right\}^{p}}{2^{p}}}{C_{N J}^{(p)}(X)} \geq \frac{1+\left(\frac{M(X)}{2}\right)^{p}}{C_{N J}^{(p)}(X)}
$$

Corollary 3.5. If the inequality $C_{N J}^{(p)}(X)<1+\left(\frac{M(X)}{2}\right)^{p}$ holds, then the Banach space $X$ has normal structure.

\section{The Generalized von Neumann-Jordan CONSTANT AND Uniform NORMAL STRUCTURE}

$l_{\infty}(X)$ denotes that the subspace of the product space $\Pi_{n \in \mathbb{N}} X$ equipped with the norm $\left\|\left(x_{n}\right)\right\|:=\sup _{n \in \mathbb{N}}\left\|x_{n}\right\|<\infty$. Let $\mathcal{U}$ be an ultrafilter on $\mathbb{N}$ and let

$$
N_{\mathcal{U}}=\left\{\left(x_{n}\right) \in l_{\infty}(X): \lim _{\mathcal{U}}\left\|x_{n}\right\|=0\right\} .
$$

The ultrapower of $X$, denoted by $\tilde{X}$, is the quotient space $l_{\infty}(X) / N_{\mathcal{U}}$ equipped with the quotient norm, and $\left(\tilde{x}_{n}\right)$ denotes the elements of the ultrapower. Note that if $\mathcal{U}$ is non-trivial, then $X$ can be embedded into $\tilde{X}$ isometrically. It was proved that if $X$ is super-reflexive, that is $\tilde{X}^{*}=(\tilde{X})^{*}$, then $X$ has uniform normal 
structure if and only if $\tilde{X}$ has normal structure [8]. We show a theorem about uniform normal structure.

Theorem 4.1. Let $X$ be a Banach space and the inequality

$$
C_{N J}^{(p)}(X)<\frac{\left(\sqrt{4+t^{2}}+t\right)^{p}}{2^{2 p-2}\left(1+t^{p}\right)}
$$

holds for some $t \in(0,1]$. Then $X$ has uniform normal structure.

Proof. Since $X$ is uniformly non-square, so $X$ is super-reflexive(see [13]), and consequently it is enough to show that $X$ has normal structure. Suppose that $X$ lacks normal structure. Then by Lemma 2 in [12], there exist $\tilde{x}_{1}, \tilde{x}_{2}, \tilde{x}_{3} \in S_{\tilde{X}}$ and $\tilde{f}_{1}, \tilde{f}_{2}, \tilde{f}_{3} \in S_{\tilde{X}^{*}}$ satisfying:

(1) $\left\|\tilde{x}_{i}-\tilde{x}_{j}\right\|=1$ and $\tilde{f}_{i}\left(\tilde{x}_{j}\right)=0$ for all $i \neq j$.

(2) $\tilde{f}_{i}\left(\tilde{x}_{i}\right)=1$ for $i=1,2,3$.

(3) $\left\|\tilde{x}_{3}-\left(\tilde{x}_{2}+\tilde{x}_{1}\right)\right\| \geq\left\|\tilde{x}_{2}+\tilde{x}_{1}\right\|$.

Let $h(t):=\frac{\left(2-t+\sqrt{4+t^{2}}\right)}{2}$ and we consider three possible cases.

Case 1: If $\left\|\tilde{x}_{1}+\tilde{x}_{2}\right\| \leq h(t)$. Let $\tilde{x}=\tilde{x}_{1}-\tilde{x}_{2}$ and $\tilde{y}=\frac{\left(\tilde{x}_{1}+\tilde{x}_{2}\right)}{h(t)}$. Then $\tilde{x}, \tilde{y} \in B_{\tilde{X}}$, and

$$
\begin{aligned}
\|\tilde{x}+t \tilde{y}\| & =\left\|\left(1+\left(\frac{t}{h(t)}\right)\right) \tilde{x}_{1}-\left(1-\left(\frac{t}{h(t)}\right)\right) \tilde{x}_{2}\right\| \\
& \geq\left(1+\left(\frac{t}{h(t)}\right)\right) \tilde{f}_{1}\left(\tilde{x}_{1}\right)-\left(1-\left(\frac{t}{h(t)}\right)\right) \tilde{f}_{1}\left(\tilde{x}_{2}\right) \\
& =1+\left(\frac{t}{h(t)}\right),
\end{aligned}
$$

as well as

$$
\begin{aligned}
\|\tilde{x}-t \tilde{y}\| & =\left\|\left(1+\left(\frac{t}{h(t)}\right)\right) \tilde{x}_{2}-\left(1-\left(\frac{t}{h(t)}\right)\right) \tilde{x}_{1}\right\| \\
& \geq\left(1+\left(\frac{t}{h(t)}\right)\right) \tilde{f}_{2}\left(\tilde{x}_{2}\right)-\left(1-\left(\frac{t}{h(t)}\right)\right) \tilde{f}_{2}\left(\tilde{x}_{1}\right) \\
& =1+\left(\frac{t}{h(t)}\right) .
\end{aligned}
$$

Case 2: If $\left\|\tilde{x}_{1}+\tilde{x}_{2}\right\| \geq h(t)$ and $\left\|\tilde{x}_{3}+\tilde{x}_{2}-\tilde{x}_{1}\right\| \leq h(t)$. Let $\tilde{x}=\tilde{x}_{2}-\tilde{x}_{3}$ and $\tilde{y}=\frac{\left(\tilde{x}_{3}+\tilde{x}_{2}-\tilde{x}_{1}\right)}{h(t)}$. Then $\tilde{x}, \tilde{y} \in B_{\tilde{X}}$, and

$$
\begin{aligned}
\|\tilde{x}+t \tilde{y}\| & =\left\|\left(1+\left(\frac{t}{h(t)}\right)\right) \tilde{x}_{2}-\left(1-\left(\frac{t}{h(t)}\right)\right) \tilde{x}_{3}-\left(\frac{t}{h(t)}\right) \tilde{x}_{1}\right\| \\
& \left.\geq\left(1+\left(\frac{t}{h(t)}\right)\right) \tilde{f}_{2}\left(\tilde{x}_{2}\right)-\left(1-\left(\frac{t}{h(t)}\right)\right) \tilde{f}_{2}\left(\tilde{x}_{3}\right)-\left(\frac{t}{h(t)}\right)\right) \tilde{f}_{2}\left(\tilde{x}_{1}\right) \\
& =1+\left(\frac{t}{h(t)}\right)
\end{aligned}
$$


as well as

$$
\begin{aligned}
\|\tilde{x}-t \tilde{y}\| & =\left\|\left(1+\left(\frac{t}{h(t)}\right)\right) \tilde{x}_{3}-\left(1-\left(\frac{t}{h(t)}\right)\right) \tilde{x}_{2}-\left(\frac{t}{h(t)}\right) \tilde{x}_{1}\right\| \\
& \left.\geq\left(1+\left(\frac{t}{h(t)}\right)\right) \tilde{f}_{3}\left(\tilde{x}_{3}\right)-\left(1-\left(\frac{t}{h(t)}\right)\right) \tilde{f}_{3}\left(\tilde{x}_{2}\right)-\left(\frac{t}{h(t)}\right)\right) \tilde{f}_{3}\left(\tilde{x}_{1}\right) \\
& =1+\left(\frac{t}{h(t)}\right) .
\end{aligned}
$$

Case 3: If $\left\|\tilde{x}_{1}+\tilde{x}_{2}\right\| \geq h(t)$ and $\left\|\tilde{x}_{3}+\tilde{x}_{2}-\tilde{x}_{1}\right\| \geq h(t)$. Let $\tilde{x}=\tilde{x}_{3}-\tilde{x}_{1}$ and $\tilde{y}=\tilde{x}_{2}$. Then $\tilde{x}, \tilde{y} \in B_{\tilde{X}}$, and

$$
\begin{aligned}
\|\tilde{x}+t \tilde{y}\| & =\left\|\tilde{x}_{3}+t \tilde{x}_{2}-\tilde{x}_{1}\right\| \\
& \geq\left\|\tilde{x}_{3}+\tilde{x}_{2}-\tilde{x}_{1}\right\|-(1-t) \\
& \geq h(t)+t-1,
\end{aligned}
$$

as well as

$$
\begin{aligned}
\|\tilde{x}-t \tilde{y}\| & =\left\|\tilde{x}_{3}-\left(t \tilde{x}_{2}+\tilde{x}_{1}\right)\right\| \\
& \geq\left\|\tilde{x}_{3}-\left(\tilde{x}_{2}+\tilde{x}_{1}\right)\right\|-(1-t) \\
& \geq h(t)+t-1 .
\end{aligned}
$$

Then, by the definition of $C_{N J}^{(p)}(X)$ and $C_{N J}^{(p)}(X)=C_{N J}^{(p)}(\tilde{X})$, we obtain

$$
\begin{aligned}
C_{N J}^{(p)}(X) & \geq \max \left\{\frac{\left(1+\frac{t}{h(t)}\right)}{2^{p-2}\left(1+t^{p}\right)}, \frac{(h(t)+t-1)^{p}}{2^{p-2}\left(1+t^{p}\right)}\right\} \\
& =\frac{\left(\sqrt{4+t^{2}}+t\right)^{p}}{2^{2 p-2}\left(1+t^{p}\right)} .
\end{aligned}
$$

This is a contradiction and thus the proof is complete.

Acknowledgement. This work was supported by Natural Science Foundation of Heilongjiang Province (A2015018).

\section{REFERENCES}

1. J. Ayerbe-Toledano, TD. Benavides and G. López-Acedo, Measures of noncompactness in metric fixed point theory, Operator Theory: Advances and Applications, 99 (1977), Birkhäuser Verlag, Basel.

2. TD. Benavides, A geometrical coefficient implying the fixed point property and stability results, Houston J. Math. 22 (1996), no.4, 835-849.

3. W. Bynum, Normal structure coefficients for Banach spaces, Pacific J. Math 86 (1980), no. $2,427-436$.

4. Y. Cui, W. Huang, H. Hudzik and R. Kaczmarek, Generalized von Neumann-Jordan constant and its relationship to the fixed point property, Fixed Point Theory Appl. 2015 (2015), no. $1,2015: 40$.

5. J. García-Falset, Stability and fixed points for nonexpansive mapping, Houston J. Math. 20 (1994), no. 3, 495-505.

6. J. García-Falset, E. Llorens-Fuster and E. Mazcuñán-Navarro, Uniformly nonsquare Banach spaces have the fixed point property for nonexpansive mappings, J. Funct. Anal. 233 (2006), no. 72, 494-514.

7. K. Goebel and W.Kirk, Topics in Metric Fixed Point Theory Cambridge University Press. 1990. 
8. MA. Khamsi, Uniform smoothness implies super-normal structure property, Nonlinear Anal. 19 (1992), no. 11, 1063-1069.

9. W.A. Kirk, A fixed point theorem for mapping which do not increase distances, Amer. Math. Monthly. 72 (1965), no. 9, 1004-1006.

10. J. Lindenstrauss, On the modulus of smoothness and divergent series in Banach spaces, Mich. Math. J. 10 (1963), no. 73, 241-252.

11. EM. Mazcuñán-Navarro, Banach space properties sufficient for normal structure, J. Math. Anal. Appl. 337 (2008), no. 1, 197-218.

12. S. Saejung, Sufficient conditions for uniform normal structure of Banach spaces and their duals, J. Math. Anal. Appl. 330 (2007), no. 1, 597-604.

13. Z. Zuo and Y. Cui, A coefficient related to some geometrical properties of Banach space, J. Inequal. Appl. 2009 (2009), no. 1, 934321.

1 Department of Mathematics, Harbin Institute of Technology, Harbin, 150001 , CHINA.

E-mail address: wangxipuremath@gmail.com; zcp@hit.edu.cn

2 Department of Mathematics, Harbin University of Science and Technology, Harbin, 150080, China.

E-mail address: cuiya@hrbust.edu.cn 